\title{
Multimodal Discourse Analysis of Digital Marketing Contents on Facebook
}

\author{
Ajepe, Ife Fiyinfolu* \\ Department of English \& Languages AfeBabalola University Ado -Ekiti, Ekiti, Nigeria
}

*Corresponding Authors: Ajepe, Ife Fiyinfolu, Department of English \& Languages AfeBabalola University Ado-Ekiti, Ekiti, Nigeria

\begin{abstract}
Multimodal discourse analysis is a framework that dissects communication in all its modes. This present study investigates two Ads from Facebook to demonstrate how linguistic and non-linguistic semiotic resources contribute to overall meaning making. The study applies Kress and Van Leeuwen's theory on Visual Grammar to analyze certain digital marketing contents. The tools employed elaborates that communication and information thrives on various semiotic modes. The findings explicated that semiotics such as color, symbols, gaze, distance, frames, etc. heightens the linguistic component of the ads.
\end{abstract}

Keywords: Multimodal Discourse, Communication, Visual Grammar, Modes, Multimodality, Semiotics.

\section{INTRODUCTION}

Communication is the basis of every human life and interaction; and it is quite important for human survival. Social interaction blooms on people's awareness of each other; and common understanding established when information is transmitted. With technological invasion and advancement, communication has moved from the traditional outlook of either face-to-face or strictly verbal message to encompass visual resources such as colors, images, designs, etc. which were initially perceived as means of entertainment. In today's societal set up, communication is enriched with the use of visual resources to heighten the means of encoding and decoding messages and the message itself. In other words, modern technology has barred the excessive dependence on verbal expression in meaning making and dented the belief that verbal mode is superior to other modes in communication as it now accommodates multiple modes alongside verbal resources in convening and interpreting codes.

Discourse analysis has equally developed over the course of time from its predominant focus on language to incorporate the scrutiny of different modes employed in the interpretation of human communication. Initially, heavy attention was paid to how language naturally occurred as connected speech whether in written or spoken discourse. Today, reference to discourse analysis now integrates the analysis of linguistic and non-linguistic systems which includes language, gestures, images, sounds, formats, etc. This is recognized as Multimodal Discourse Analysis.

Multimodal Discourse Analysis (henceforth, MDA) is a sub-field of Discourse Analysis that has garnered prominence as of late based on the pioneering works of Kress and Van Leeuwen. They argued that images are linguistic forms, that is, as words are verbal language so also are images, visual language. Thus MDA is in the business of meaning-making through the combination and the integration of multiple modes of communication. MDA explores how multiple resources such as text, image, sound, color, gesture, font, etc. are combined to transmit information or to communicate. According to Adama (2016), "multimodality in its most fundamental sense is the coexistence of more than one semiotic mode within a given context". Multimodal discourse analysis therefore, is the fusion of interdependent resources for semantic implications. 


\section{RESEARCH QUESTION AND OBJECTIVE}

This paper aims at investigating the interaction between the linguistic and non-linguistic resources employed in two Facebook Digital marketing ads to ascertain how these ads convey meanings using Kress and Van Leeuwen's Visual Grammar theoretical framework.

The paper seeks to address the following questions:

1. What are the linguistic resources and non-linguistic resources that can be identified and analyzed in the selected Ads?

2. How are the participants and viewers in the selected ads represented?

3. In what ways do the identified and analyzed linguisticand non-linguistic resources contribute to communication?

4. In what ways do the participants and viewers' representation contribute to meaning making?

5. Which visual communication modes are most salient in the selected ads?

The channels of communication may be through the television, the radio, internet, telephone, paintings, drawings, sculptures, newspapers and magazines. For example, the television displays visual forms in our houses, the radio transmits sounds in our vehicles, the telephone buzzes in our ear drums, paintings hang on our hallways, drawings in our classrooms, sculptures and fashion galleries in all our streets, the newspaper on our desks and the magazines in our libraries.

All these channels display all kinds of communication. The internet has attracted a spectrum of attention being a channel that implements multimodality. The internet consists of diverse forms such as static pages, online interaction platforms such as Facebook, WhatsApp, Instagram, Snapchat, Zoom, etc. These online platforms perform many functions such as interaction, followership, publications, etc. and due to the ever increasing participants, organizations are beginning to see the importance of these online platforms and thus making up strategies with which they can maximize it.

One of the means by which organizations maximize these online platforms is advertising. Dyer (1982) posits that an advertisement is a message that is aimed at persuading readers or listeners to buy a particular product, favor a particular organization or agree with a particular idea. McDonald (1992) likewise observes that advertisements are all around us: in our daily newspapers, magazines, on boardings in the street, on buses and assailing us from the radios, televisions and the cinema. Each of these means of advertising has what the advertiser relies on most for effective communication which includes both linguistic and semiotic resources. In other words, various multimodal resources are combined when placing advertisement whether in print or on social media.

Right from the onset, MDA according to Mach in (2007) is "a social semiotic approach to visual communication.... which provides a tool kit for the analysis of visual compositions such as advertisements, magazines pages and covers, photographs, school books, and web pages, etcetera." MDA is the parameter for pinpointing and understanding the positioning of many semiotic modes and how they are synchronized to generatea unified text or communication. Due to the growing necessity to scrutinize meaning beyond language means in several purviews of contemporary writing, MDA explains textual structure, not by single mode of composition, but visually through layout, color, and typography both at the level of text and that of discourse (Liu, 2013).

In MDA, there is an extensive array of elements engaged to illustrate how visual compositions Care produced from a set of obtainable selections using means that are ruled by conventions. Some of these elements according to Mach in (2007) are:

\subsection{Denotation}

In visual composition, denotation signifies the real depiction of an image in the physical world, that is, what an image represents in reality. Our world knowledge is a strong determining factor of the interpretation of what we see in any image. This background knowledge helps us to generate meaning "rather than just seeing." Thus, denoting people in particular places or in groups, from different angles, in distance or nearby will have an effect on how we see them. 


\subsection{Connotation}

In visual composition, connotation is an ancillary semiotic resource, which Crystal (1992) refers to as "all kinds of association a word or substance may evoke, whether emotional, or situational, particularly in certain contexts over and above denotation." Mach in (2007) opines that "asking what an image connotes is asking: what ideas and values are communicated through what is represented."

Thus, for an inclusive study of an image at this stage, elements that project meaning from other domains must be expressively considered. Drawing from Barthes (1977), there are numerous styles of representation that are actual carriers of connotation. They are:

Pose: This includes several standing, sitting or lying postures. These postures can be used to suggest different gestures associated with different emotions such as aggression, determination, confidence, satisfaction, etc.

Objects: These are props or materials used in the images. These objects have the ability to carry meanings contingent on how they are placed and their use amidst other props.

Settings: These refers to space, panorama, scenery, background or the surrounding in which something is set.

\subsection{Degrees of Articulation Of Background}

These refer to the variations of backgrounds oscillating from a blank space, through a blithely outlined in-focus, slightly out-of-focus or completely out-of-focus/faded to maximally sharp and detailed background. Depending on which one is used, every background speaks about the images. For instance, where nothing can be said about the background of the image, it means that such image is symbolic and therefore decontextualised.

\subsection{Degrees Of Color Saturation}

It is concerned with the true nature of colors; how it appears ranging from black and white to maximally saturated colors. Saturated colors make images more than real and it increases the sensuousness or emotional engagement of advertisements.

\subsection{Color Differentiation}

This has to do with numbers of colors used in images. It is the scale from maximally diverse, to monochrome which is done in a range of tones of single color.

\subsection{Semiotics of Colour}

Color has two kinds of value based on the observation made by Kandinsky (1997). One of these values is "direct value" which has to do with the effect that color has on the viewer. The other value is the "associative value" and it is concerned with the convention or certain meaning qualities that have been associated with particular colors.

However, Kress and Van Leeuwen drawing on Halliday's SFG theory came up with one suitable for the analysis of color in visual design.

Ideational Function: Color is used to convey ideas in everyday contexts. It can denote specific people, places and things as well as their classes. For this reason, color is analyzed based on how it appeals to the knowledge of the people in the real world.

Interpersonal Function: Color is analyzed in terms of how it relates with the audience. According to Mach in (2007), he says that the point of analysis here is not concerned with a lexis use of semiotic resources but the way their meaning potential is realized in communicative contexts.

Textual Function: Color is analyzed here in terms of its coherence. It indicates the same order of things. In advertisement for example, the same colour may be used on a product, on part of accompanying picture and in the fonts for the slogan and information.

\subsection{Visual Participants}

Participants in visual communication may be represented as individuals and groups categorization. There is also an instance of non-representation which deals with a situation where certain categories of people are not represented in pictures of settings where they are actually present. 


\subsection{Viewers}

Gaze: No matter the positioning, what is relevant here is how the images are designed to communicate with the readers or viewers. Kress and Van Leeuwen (1996) states that “...we can think about images in terms of the two image acts 'offer' and 'demand'. And we can also think about them in terms of expected responses." (Mach in 2007). So, if the participant is designed to look at the viewer, this means that it creates a visual form of address thereby acknowledging the readers or viewers. Where the represented participants do not look at the viewer on the other hand it means that there is no contact made with the viewer and no demand of them.

Angle of Interaction: This has to do with the angle from which we view participants. It can suggest different relations between the people represented and the viewer just as in real life. Angle of interaction can be horizontal where viewers meet the participants face-to-face or the image looking off frame (not designed looking in the direction of the readers or viewers). It can also be vertical where it will be embedded with semiotic of power and the association of height and superiority/inferiority.

Distance: This signifies social relations; it translates as 'size of frame' ranging from close shot, medium shot, long shot, etc. It is associated with physical proximity and intimacy.

\section{AnAlysis, Results, Findings ANd Discussion OF Findings}

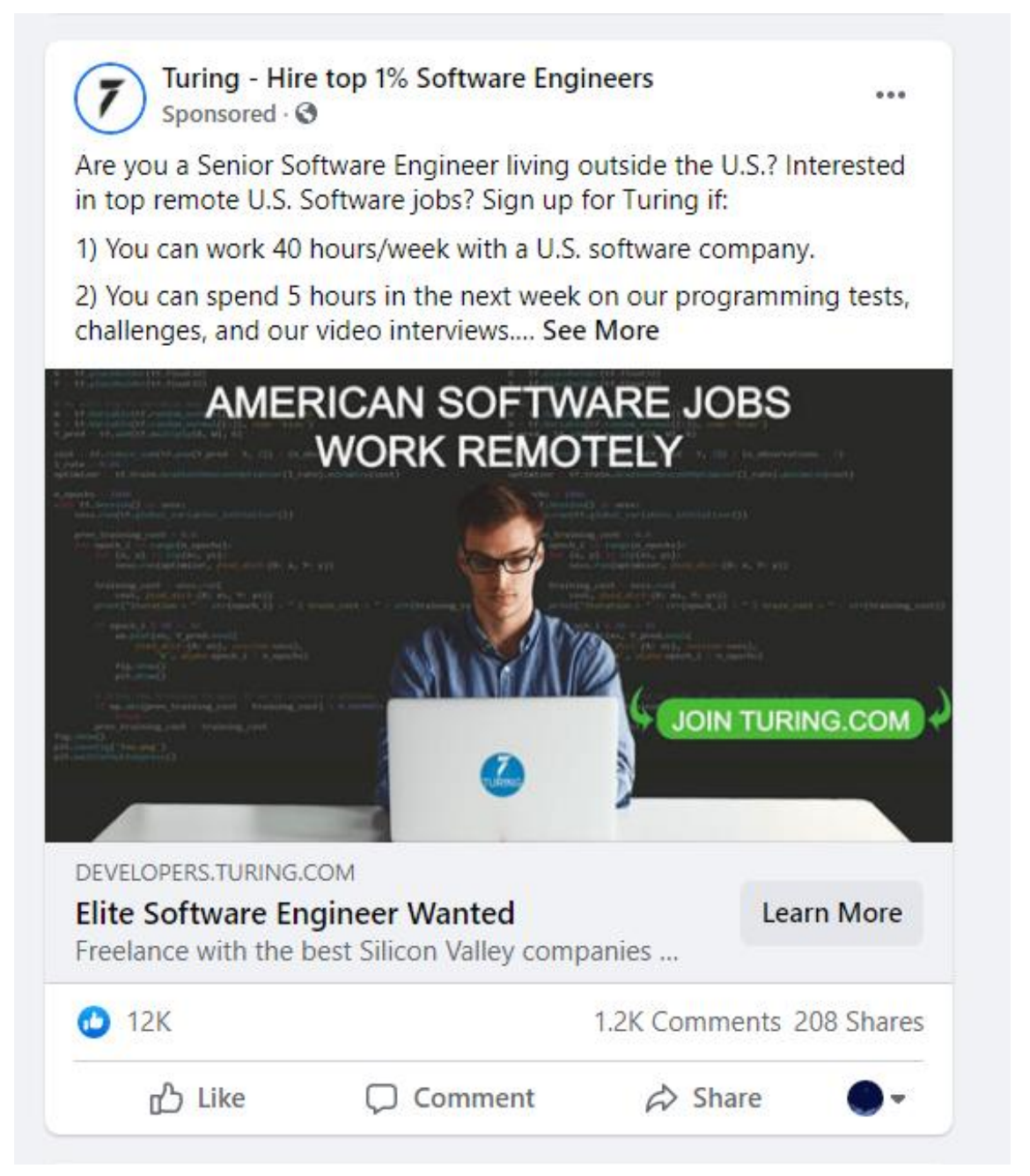

There are three dimensions to the image above. They are: the man, the background picture and the text. All the items on display constitute visual elements starting from the participant in the image. The category of visual participants that can be obtained from this image is one which is technically known as individual. This is because in visual composition, according to Mach in (2007), individualization is realized by shots that show only one person.

In this image, there is a Caucasian male in a sitting pose, dressed in blue shirt and wearing glasses operating on a laptop bearing the logo of the company being advertised. The pose of the participant suggests an operational comfort in the tool being handled 
The man's blue shirt which is unbuttoned at the collar suggests that the man is at home in the task at hand while depicting a professional and artistic sensitivity. The glasses also lend a scholarly composition to the individual. The computer, which is front and centre; and the blackboard, which is the background picture serves as props which heightens the message being passed to the viewers.

The image shows an individual who finds a balance between being corporately and comfortably dressed and given a medium close shot, this would suggest the viewers' identification with the participant in terms of the general idea or belief that people who dress in unbuttoned shirts are often formal, friendly and accessible. The use of a Caucasian male likewise suggests that the work being offered is American engineered as attested to by the text "AMERICAN SOFTWARE JOBS WORK REMOTELY".

The gaze of the participant is likewise directed at the object in front of him rather than at the responder. This gaze stance is known as offer which encourages the viewer to pay close attention to the object that has captured the attention of the visual participant. The image is likewise centred-frame which is more symbolic in that it urges the viewers to serve as an observer to the benefit of what is being offered with Turing's employment.

There are four noticeable colors in the image: blue, green, white and black. The visual participant is dressed in blue which is the color of the company's logo. Blue is a serene color that represents tranquility, composure, dependence, veracity, rectitude, intelligence and responsibility. By making participant's shirt blue, Turing identifies with the participant. In the same vein, what is presented to the viewers is a company that is trustworthy, serene and that has workers that are composed, dependable, responsible and intelligent. This does invites the viewers to be a part of this brand by seeking employment with them. Green symbolizes tranquility, black symbolizes faith and trust while black represents strength and authority. All which are necessary endorsement for a company.

The background image features a chalkboard with computer gibberish. This symbolizes authority in the software engineering field and points the viewers' attention to innovation in technology. Worthy of note here is how the background is subtly blurred to give room for focus on the male (participant).

The image also presents a sense of organizational standard to the potential employees' sub consciousness even before they engage actively the textual content. The text also reinforce the information intended, that only clientele with such carriage and charisma are required to offer their services in the organization.

The angle and distance of interaction with the viewers is horizontal and close shot respectively. This is associated with close proximity and intimacy which is needed between an employer and potential employee.

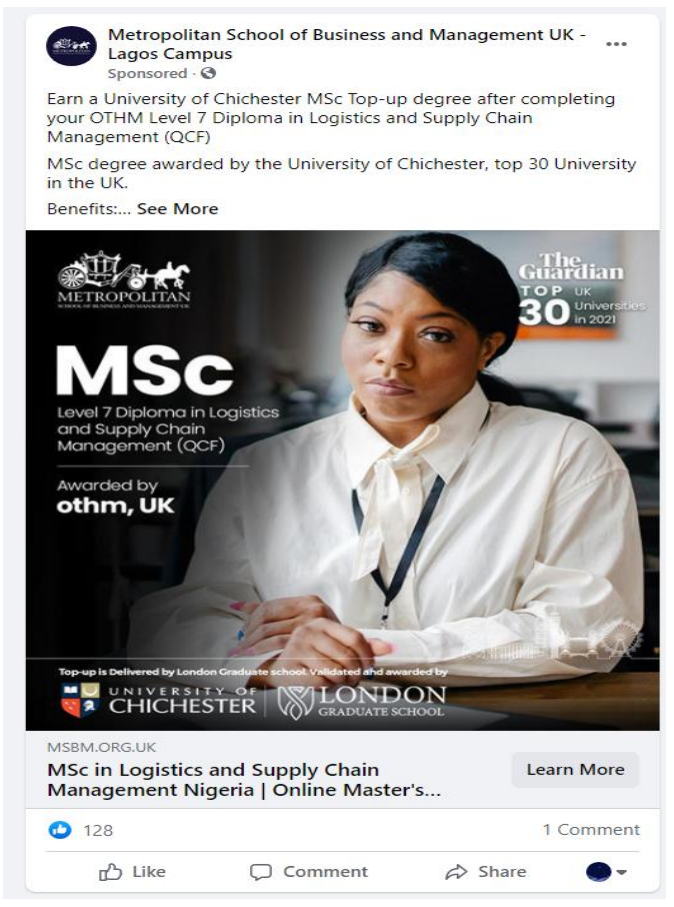


The African woman in the image above is technically known as an individual. The use of a woman of color as the visual participant of this image foregrounds several social issues such as the ease of acquiring a degree from a foreign university while systematically studying for it in your own country, the empowerment of the girl-child, the possibility of beauty with brains regardless of what society says, etc.

In the image, the African female is in a sitting pose, dressed in a grey buttoned up shirt with an ash suit top hanging at the back of her seat. These colors suggest softness, complexity, fluidity and technical know-how. Ash and gray are likewise the colors of intellect, strength, formality and diplomacy. All which are salient characteristics of the academia. They are cool and neutral colors that suggest that products of the university been advertised are formal, resilient, modern and sophisticated. Thus her pose shows a high level of seriousness and professionalism which is the nature of academics while paying equal attention to fashion.

In this image, the participant gazes directly at the viewers. This gaze unswervingly demands the attention of the viewers and creates a kind of symbolic contact or interaction between the viewer and the image depicted. The table, white paper and chair serves as props in the image. The background picture also heightens the message being passed to the viewers of the seriousness and formality of the situation at hand- learning.

The logo which is black, white and grey is made up of a chariot with a cart that is mobile, which depicts upward progress. The color black represents strength, seriousness, power, and authority that come with maximizing that which the university has to offer its targeted audience. The color white, in heraldry, depicts faith and purity which is needed to garner participation of the viewers.

The angle and distance of interaction with the viewers is horizontal and close shot respectively. This is associated with close proximity and intimacy.

\subsection{Findings}

This paper has scrutinized how linguistics and non-linguistics resources reinforce communication. The digital marketing tools show how firms portray their intention to potential customers to gain clientele. The data is analyzed through the qualitative method, which is inductive. The method helped in attaining the desired result of meeting the needs of the target audience that is the readers of the Facebook commodity. This aided the ability to give a vivid description and interpretation of the data.

The non-linguistic resources in the image takes both forms of offer and demand. They are usually expressed as being involved in one action or the other. These actions depict the message that is being passed across by the firms. The images in the digital marketing tools present an explanation of the message being passed across in the Facebook Advertisement. This is a good strategy employed by the organization in providing information to the readers. The non-linguistic resources used portray this purpose of giving information not demanding information.

Through the interaction of the linguistic and non-linguistic resources, it has been discovered that the non-linguistic resources do more explanation than the linguistic resources. The linguistic resources are nevertheless used to express the actions depicted through the non-linguistic resources. The nonlinguistic resources are scattered everywhere and their presence was a major formation in the general makeup of the meanings intended.

Another observation is that there is interaction between the non-linguistic resources and the linguistic resources. This shows a connection between the two resources. From the findings above, it can be clearly stated that there is coherence between the two resources and their placement

\section{CONClusion}

The research has succeeded in reducing the paucity of knowledge on the application of multimodal discourse analysis to investigate the meaning- making of linguistic and nonlinguistic resources on any given visual texts. How various semiotic resources are combined to make meaning has been thoroughly investigated so that there may be more effectual and comprehensive attachment of meaning given to any visual texts. In all, how linguistics and nonlinguistic resources, which have been identified and analyzed, interact in conveying meaning has been investigated.

We can conclude that the result and analysis of the investigation of the research can help in dishing out effective recommendation on the various linguistic and non-linguistic resources which can be combined in establishing attractive advertisement on any magazine or visual texts. 


\section{REFERENCES}

[1] Adama, E. (2016). Linguistic Landscape. In Jewitt, C., Bezemer, J., \& O’Halloran, k. (Eds) Introducing Multimodality (1st ed., pp.451-459). Abingdon, Oxon: Routledge

[2] Barthes R. (1977), Image, music,text. In S.Heath (Ed.) the Journal of Aesthetics and Art Criticism 37 (2): 235 - 236. NewYork: Hill and Wang.

[3] Crystal D. (1992) Introducing Linguistics. Harmondsworth: Penguin.

[4] Dyer, G. (1982). Advertising as communication. London: Routledge.

[5] Kress, G. R., \&Van, L. T. (2006). Reading images: The grammar of visual design. London: Routledge.

[6] Liu, J. (2013). Visual images interpretive strategies in multimodal texts. Journal of Language Teaching and Research. 4(6) pp. 1259-1263. Finland: Academy Publisher.

[7] Machin, D. (2007). Introduction to multimodal analysis. New York: Hodder Arnold.

[8] McDonald, C. (1992) How Advertising Works. London: The Advertising Association and NTC.

\section{AUTHOR'S BIOGRAPHY}

Ife Fiyinfolu Ajepe, holds a Ph.D in English Language, with specialization in Multimodal Discourse Analysis and Communication. She has interests in Semiotics, and Applied linguistics. She is a Senior lecturer with over ten years' teaching experience. She currently works with AfeBabalola University, Ado - Ekiti, Nigeria. She is married with children.

Citation: Ajepe, Ife Fiyinfolu. "Multimodal Discourse Analysis of Digital Marketing Contents on Facebook" International Journal on Studies in English Language and Literature (IJSELL), vol 9, no. 8, 2021, pp. 1-7. doi: https://doi.org/10.20431/2347-3134.0908001.

Copyright: (C) 2021 Authors. This is an open-access article distributed under the terms of the Creative Commons Attribution License, which permits unrestricted use, distribution, and reproduction in any medium, provided the original author and source are credited. 